\title{
Simulation and Research on Response Characteristic of Optical Fiber Probe Sensor of Void Fraction Measurement Based on Zemax
}

\author{
Lingfu Kong \\ College of Information Science and Engineering \\ Yanshan University \\ Qinhuangdao, Hebei 066004, China \\ lfkong@ysu.edu.cn \\ Weihang Kong \\ College of Information Science and Engineering \\ Yanshan University \\ Qinhuangdao, Hebei 066004, China \\ kongweihang@163.com
}

\author{
Na Xie \\ Information Department of Library \\ Yanshan University \\ Qinhuangdao, Hebei 066004, China \\ xienahaoyun@163.com \\ Yingwei Li \\ College of Information Science and Engineering \\ Yanshan University \\ Qinhuangdao, Hebei 066004, China \\ lyw@ysu.edu.cn
}

\begin{abstract}
In view of the void fraction measurement problem of oil-gas-water three phase flow in oil production logging, in this thesis, optical fiber probe sensor is used to measure the cross-sectional local void fraction. In order to analysis post-treatment raw probe signal preferably and more reasonable, firstly, the characteristic of optical fiber probe pierced bubble is analyzed and simulated by the simulation modeling with ZEMAX, and adopting nonsequential ray tracing method to simulate the track characteristic of the light energy variation in the sensing system, which both from the different horizontal radial positions of probe pierced bubble and different axial positions of the probe; Then, in order to get the accurate local void fraction information, gas-phase distribution features in fluid pipeline and flow regime identification of multiphase flow, the transformation of binary value signal in probe signal post-processing is analyzed and compared by different threshold methods. Finally, the experimental platform of void fraction measurement is built to obtain the probe raw signal of oil-gas-water three-phase which total flow is $30 \mathrm{~m}^{3} / \mathrm{d}$, and the effects of different threshold method are confirmed.
\end{abstract}

Keywords: Optical fiber probe sensor; Void fraction; Response characteristic; Signal threshold processing; Zemax simulation

\section{INTRODUCTION}

At present, most of oil deposits in China have entered into the stage of middle-late mining, existing a large number of formation water in reservoir, and along with the phenomenon of degassing in the oil layer, which leads to a mixed flow characteristic of oil-gas-water three-phase flow. The intervention of gas phase affects the flow characteristic of three-phase flow to a large extent, which makes the complex flow condition of oil-gas-water three phase flow in oil wells, results in the flow pattern, pressure, flow velocity, flow direction and other factors of three-phase flow are closely related with it, and brings great difficulties to detect the parameters [1-4]. Due to the physical character differences like density, viscosity, and slippage velocity between the gas phase and liquid phase, that shows the different output response signals of optical fiber probe sensor. Therefore, in order to further analyze the response characteristics of probe pierced bubble in the complex oil-gas-water three phase flow, we need to deeply understand the mechanism of probe pierced bubble, and qualitatively analyze the response character-istics of the probe influenced by various situations of puncture bubble. Also, it provides a solid theoretical foundation for optimization of sensor, signal processing, etc.

The response characteristic of optical fiber probe pierced bubble is influenced by the gas and liquid surface tension, bubble size and shape, the pierced bubble position and sensor system noise and so on, results in the response signal waveform of optical fiber probe is a non-ideal highlow level signal. Therefore, the reasonable selection of gas-liquid phase transition threshold algorithm is crucial. Tang renhu et al [5] made a systematic research and summary on measuring a local void fraction by probe method, and pointed out that signal post-processing is the key of measuring void fraction. Angeli et al [6] adopted the automatic adjustment dual-threshold method for processing signal, which proposed by Van Der Welle R et al [7], and achieved better results. Sakamoto A et al [8] and Yang Sheng et al [9] employed a single threshold method to distinguish the signal, which belongs to gas or liquid phase for processing signal, and calculated the averaged void fraction.

In summary, in order to make the measuring signal a better reflection of the parameter characteristics about oilgas-water three-phase flow, in this thesis, the response characteristics and mechanism of probe pierced bubble are 
further analyzed, to obtain a desired void fraction of oilgas-water three phase flow, to provide an important basis for the dynamic monitoring analysis of oil production logging.

\section{THE PRINCIPLE OF OPTICAL FIBER PROBE MEASURING}

Optical fiber probe method [10] is based on the principle of total reflection, using a different refractive of gas-liquid phase media index to light with gas-liquid fluid through fiber-optic probe alternately, converted to a continuous change in high and low level of voltage signal by detecting the reflected light intensity, and got the fiber optic probe partial sectional void fraction. When the optical fiber sensitive probe in contact with the gas-phase, as shown in Figure 1(a), the incident light occurs to the total reflection in the sensitive probe, and outputs a highlevel signal; when the sensitive probe in contact with the liquid, as shown in Figure 1(b), the incident light mostly occurs to refraction in the sensitive probe, and outputs a low-level signal.
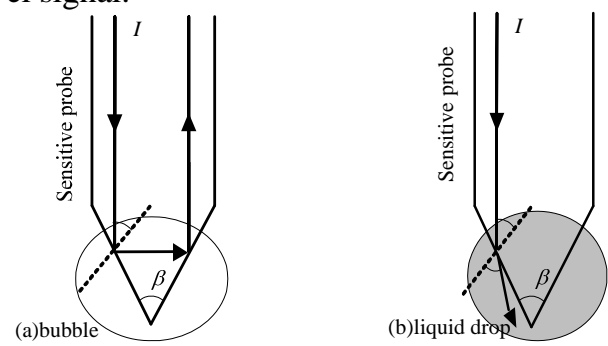

Figure 1. Optical fiber probe to measure the gas-liquid phase schematic

\section{THE RESPONSE CHARACTERISTICS OF OPTICAL FIBER PROBE PIERCED BUBBLE}

In oil production logging environment, due to the random variation characteristics measurement of fluid, there is no regular pattern about bubble motion in measurement fluid. Furthermore, the measurement bubbles are of different sizes, different shapes, and the horizontal radial position from the center to edge by probe pierced bubble has a certain amount of random, which will cause different signal response characteristics on different horizontal radial positions of probe pierced bubble. Therefore, it plays an significant role in reasonable signal post-processing that making a further exploration and analysis on the different horizontal radial positions and response characteristics, during the whole process of probe pierced bubble. In this thesis, the method of modeling with ZEMAX is used to analysis the response characteristic of optical fiber probe pierced bubble, and adopt nonsequential ray tracing method [11] to simulate the light energy variation in the sensing system during the whole probe pierced bubble process. Thereby, the characteristic distribution of returned energy on different horizontal radial positions can be obtained.

The diagram of different horizontal radial positions of probe pierced bubble is shown in Figure 2(a). Where the spherical bubble radius is $\mathrm{R}$; the radial distance from spherical center to probe axial is $\mathrm{r}$. The Zemax simulation model of probe pierced bubble is shown in Figure 2(b). Assuming the probe sensitive head is cone, the angle is $35^{\circ}$, the material is sapphire, the radius of spherical bubble $\mathrm{R}$ is equal to $2 \mathrm{~mm}$. By means of building the analog simulation of piercing process on different radial positions $r$, processing millions of ray tracing when the probe sensitive head has reach a certain axial position every time, until the probe complete the whole process of pierced bubble. Thereby the response characteristics of different axial positions of the probe are obtained.
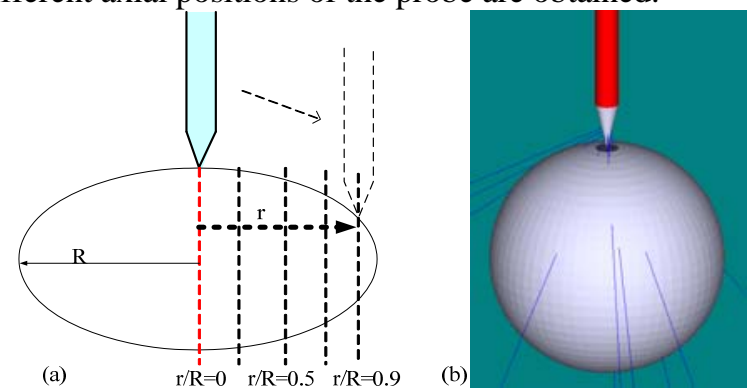

Figure 2. The diagram of different positions and Zemax simulation model of probe pierced bubble

In Fig .2(b), the response characteristic is simulated by changing the radial distance $r$ from the bubble center to probe axial, which takes the normalized pierced position $\mathrm{x}$ (equal to r/R) with 0, 0.5, 0.85 and 1, respectively. At the normalized position $\mathrm{x}$, along the Z-axis direction with an increment of $0.5 \mathrm{~mm}$ to pierce bubble, finally, the response curve distribution diagram of returned power on different $\mathrm{Z}$-axis displacement of horizontal radial position $\mathrm{x}$ with probe pierced bubble is obtained, as shown in Figure 3. Through analysis of Figure 3, we know that it can get the varies response curves of returned power in different position $\mathrm{x}$. The longest gas-phase residence time can be obtained when the probe is piercing bubble on the central position $x=0$, and the intensity of returned power is larger. With the horizontal radial position of probe pierced bubble is far away from the bubble center, getting the gas phase residence time will gradually reduced. When the probe is on the position $\mathrm{x}=1$ with the extreme edge of the bubble, not only the gas phase response residence time becomes shorter, but also the amplitude of returned power suddenly drops, meanwhile it differs for several times with pierce the bubble center position.
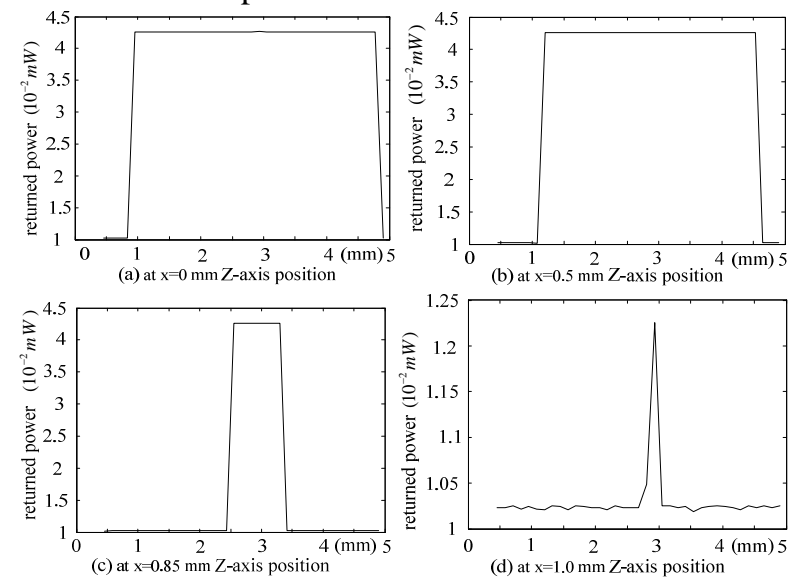

Figure 3. The response characteristic distribution diagram of returned power of probe pierced bubble in different horizontal radial positions

According to the probe measurement principle, the strength of returned power and gas-phase residence time are directly reflect the amplitude of the probe raw voltage 
signal by photoelectric conversion and the response characteristic of high level duty cycle which varies from hour to hour. Revealed by analog simulation, since the influence of gas-liquid interfacial tension and the different pierced bubble radial position of the probe tip, puncture tension of gas-liquid interface delay, results in the output signal is an irregular, instead of the regular rectangular wave, and just as the curve of returned power on different positions of the same $x$ in Fig .3. Meanwhile, the response characteristics of probe pierced bubble are obtained by Zemax simulation modeling, and directly reflect the response signal $V(t)$ of measurement void fraction of oilgas-water three phase flow by using optical fiber probe sensor, as shown in Figure 4. From the analysis of Fig .4, both the gas-phase residence time region of different sizes and signal response amplitude of different sizes are existing in the probe raw signal.

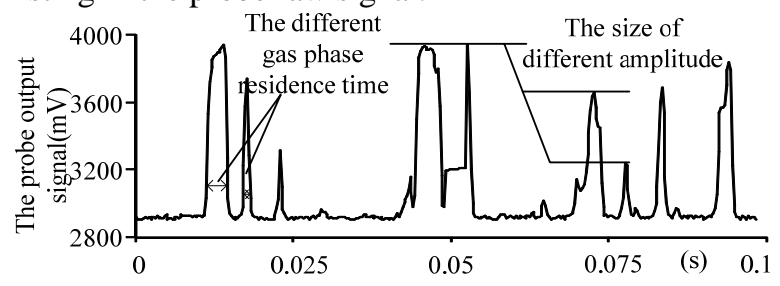

Figure 4. The raw signal of optical fiber probe

\section{THE SIGNAL THRESHOLDING OPERATION}

Due to that the response characteristic of optical fiber probe pierced bubble is influenced by gas-liquid surface tension, bubble size and shape, the pierced bubble different positions and sensor system noise, results in the output raw signal of optical fiber probe is approximate to the trapezoid or "bell" wave. According to the gas-phase time proportion of total time, which passing the probe calculate void fraction, the gas holdup measurement is calculated through time, and it is very important to increase the accurate measurement of void fraction for accurately distinguish between gas phase and liquid phase signal. Consequently, the output signal of optical fiber probe is threshold processing appropriately and responsibly, in order to accurately determine the total time which the bubbles or air mass through the probe, and finally the cross-sectional void fraction is obtained.

\section{A. Conventional min-max method}

\section{1) The single threshold method}

The principle of single threshold method refers to the probe bounded low voltage signal, only requires one threshold $V_{\text {th }}$ to process the gas-liquid phase separation, which translated into binary value signal. When the signal voltage value $V(t)$ is greater $V_{t h}$, it means the head in gas phase now, and set to 1 ; otherwise, below the threshold, it means the head in the liquid phase now, and set to 0 ; Equation (1) is:

$$
f(t)= \begin{cases}1 & , V(t)>V_{t h} \\ 0 & , V(t)<V_{t h}\end{cases}
$$

Where to make sure threshold $V_{\text {th }}$ through maximum and minimum of signal value, according to the extent experience or effect of noise to determine the parameter $p$ to get any value ranging from 0 to 1 , the $V_{\text {th }}$ is expressed as Equation (2) :

$$
V_{t h}=p \times\left(V_{\max }-V_{\min }\right)+V_{\min }
$$

It can be obtained the threshold variable $p$ by through the “(2)” and corresponding expression as Equation (3).

$$
p=\frac{V_{t h}-V_{\min }}{V_{\text {max }}-V_{\text {min }}}
$$

A unitary processing to the continuous voltage signal $V(t)$ of optical fiber probe, and it can be obtained the $V_{w}(t)$ expression (4).

$$
V_{w}(t)=\frac{V(t)-V_{\min }}{V_{\max }-V_{\min }}
$$

According to the "(3)" and “(4)", it can be known that the threshold variable with $p$ is equal to the normalized signal with $V_{w}(t)$, and the corresponding $V_{w}(t)$ change curve of void fraction is obtained by changing the size of variable threshold with $p$, as shown in Figure 5.

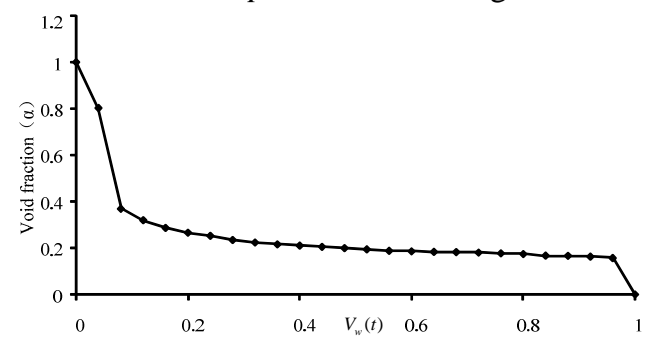

Figure 5. Void fraction change curve of normalized post-processing with signal $V_{w}(t)$

From the analysis of Fig .5, along with the $V_{w}(t)$ is larger, the overall distribution curve of void fraction appears deterioration trends. Due to the irregular signal includes the rising and falling time-delay, it brings a certain error to results. When the threshold value is fetching in 30\%-70\% with the impulse amplitude of probe output signal, gas rate tends stably, influenced less by the threshold. Therefore, the value of threshold variable with $p$ is critical, during the single processing by threshold method.

2) the fixed dual-threshold method

The fixed dual-threshold method core idea is to obtain the maximum value $V_{\max }$ and minimum value $V_{\max }$ of the $\mathrm{N}$ sampling points sequential, in processing signal with $V(t)$. Comparing the two values, according to 1 logic table, to ensure the pattern of the sample point signal. In the beginning, two initial values for $V_{\max }$ and $V_{\min }$ are given. If $V_{i}$ is greater than $V_{\max }$, then the maximum $V_{\max }$ is changed to $V_{i}$, and keep the $V_{\min }$. If $V_{i}$ is lower than $V_{\min }$, then the minimum changes to $V_{i}$, and keep the $V_{\max }$. 
If $V_{i}$ are both equal or greater than $V_{\min }$ and equal or less than $V_{\max }$, so there is no change in the maximum and the minimum values. Then the amplitude of the $\mathrm{N}$ sampling points for signal are compared with the maximum and minimum values, in this comparison, the margin $V_{H}$ and $V_{L}$ are related to the signal noise and the degree of timedelay of the signal rise or fall. If Equation (5) is true, then the output is 1 (which represents the gas phase).

$$
V_{i}>V_{\max }-V_{H}
$$

While if Equation (6) is true then the output is 0 (which represents the liquid phase).

$$
V_{i}<V_{\min }+V_{L}
$$

If neither "(5)" nor “(6)" is true, then the previous value ( 1 or 0 ) is outputted, and two values are kept. The whole signal is thus converted into a series of 1 's and 0 's, which represent each one of the two phases, it can calculate partial void fraction of the probe.

TABLE I. THE METHOD OF THE PROBE SIGNAL PROCESSING OF FIXED DUAL-THRESHOLD

\begin{tabular}{cccc}
\hline Condtion & Minimum & Maximum & Output \\
\hline$V_{i}>V_{\max }$ & $q$ & $V_{\max }=V_{i}$ & \\
$V_{\min } \leq V_{i} \leq V_{\max }$ & $q$ & $q$ & \\
$V_{n}<V_{\min }$ & $V_{\min }=V_{i}$ & $q$ & \\
$V_{i}>V_{\max }-V_{H}$, “(5)” & & & 1 \\
$V_{i}<V_{\min }+V_{L}$, "(6)” & & 0 \\
If none of “(5)” and “(6)” true & & $q$ \\
\hline
\end{tabular}

$\mathrm{q}$ : no change, the variable $\mathrm{i}$ value is range from 1 to $\mathrm{n}$.

\section{B. The method of self-adjusting dual-threshold}

A signal processing technique, based on a method of the self-adjusting dual-threshold signal processing, which proposed by van Der Welle [7], and a good result is obtained in a irregular high and low voltage signal processing. This method can detect the rise or fall location of a signal, then transforms the irregular raw signal into a rectangular wave signal, so as to obtained the partial void fraction. The core idea is that each sample of the signal is compared with two self-adjusting trigger levels and in accordance with the table comparing 2 logic table, to determine the type of the sample point signal. The signal amplitude $\alpha_{n}$ of the $n^{\text {th }}$ sample is compared with the amplitude $\alpha_{n-1}$ of the previous sample, and two adjustable maximum $\alpha_{\max }$ and minimum values $\alpha_{\min }$ respectively. In the beginning, we initial values for $\alpha_{\max }$ and $\alpha_{\min }$ are given. If $\alpha_{n}$ is greater than $\alpha_{n-1}$, then the maximum $\alpha_{\text {max }}$ is changed to $\alpha_{n}, \alpha_{\min }$ is kept. If $\alpha_{n}$ and $\alpha_{n-1}$ are equal then there is no change in the maximum and the minimum values. If $\alpha_{n}$ is lower than $\alpha_{n-1}$, then the minimum changes to $\alpha_{n}, \alpha_{\max }$ is kept. The amplitude $\alpha_{n}$ is then compared with the new maximum and minimum values. In this comparison, the margin x (signal “clip" level) associated with the signal noise.
If Equation (7) is true, then the output is 1 (which represents the gas phase).

$$
a_{n}>a_{\min }+x
$$

While if Equation (8) is true then the output is 0 (which represents the liquid phase).

$$
a_{n}<a_{\max }-x
$$

If neither "(7)” nor "(8)" is true then the previous value (1 or 0 ) is kept. The whole signal is thus converted into a series of 1 's and 0 's, which represent each one of the two phases. It can calculate partial void fraction of the probe.

TABLE II. THE SIGNAL PROCESSING METHOD OF THE SELFADJUSTING DUAL-THRESHOLD (VAN DER WELLE [7], ANGELI [6])

\begin{tabular}{cccc}
\hline Condition & Minimum & Maximum & Output \\
\hline$a_{n}>a_{n-1}$ & $q$ & $a_{\max }=a_{n}$ & \\
$a_{n}=a_{n-1}$ & $q$ & $q$ & \\
$a_{n}<a_{n-1}$ & $a_{\min }=a_{n}$ & $q$ & \\
$a_{n}>a_{\min }+x$,“(7)” & & & 1 \\
$a_{n}<a_{\max }-x$,“(8)” & & & 0 \\
If none of “(7)” and “(8)” true & & & $q$ \\
\hline
\end{tabular}

q: no change.

The statistics analysis curve of the normalized threshold value $V_{w}(t)$ is obtained by single threshold and self-adjusting dual-threshold methods, which for the probe raw signal in Fig .4, as shown in Figure 6. According to the analysis to show Fig .6, when the threshold $\mathrm{x}$ in "(7)" corresponding $V_{w}(t)$ ranges from 0.1 to 0.8 , using selfadjust dual-threshold method to get the overall stability of the mutative void fraction is better than the single threshold method, even the single threshold method come into being greatly influenced.

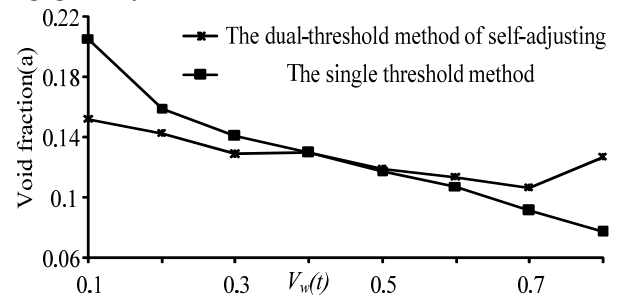

Figure 6. The curve of statistical analysis with normalization $V_{w}(t)$

\section{EXPERIMENTAL RESULTS AND ANALYSIS}

For a binary signal, sampling frequency is determined by the factors of fluid velocity and bubble diameter. This experiment, using optical fiber probe to measure the void fraction of oil-gas-water three-phase flow, in which the pipe diameter is $20 \mathrm{~mm}$ and the total fluid flow is $30 \mathrm{~m}^{3} / \mathrm{d}$, then the fluid velocity is about $1 \mathrm{~m} / \mathrm{s}$. Assuming the bubble diameter is $4 \mathrm{~mm}$, the required sampling interval cannot exceed the maximum $4 \mathrm{~ms}$, that is to say, the sampling frequency must be at least $500 \mathrm{~Hz}$. To meet the velocity and bubble diameter range, the sampling frequency is selected to be $4 \mathrm{KHz}$. The above three kinds of threshold methods are used to 2 value processing for the probe raw signal, and the signal waveform renderings, as shown in 
Figure 7. Due to that the different impact of the light with oil-water phase, the different locations of probe pierced bubble and other factors, results in the probe signal at a low level in small amplitude noise signal.
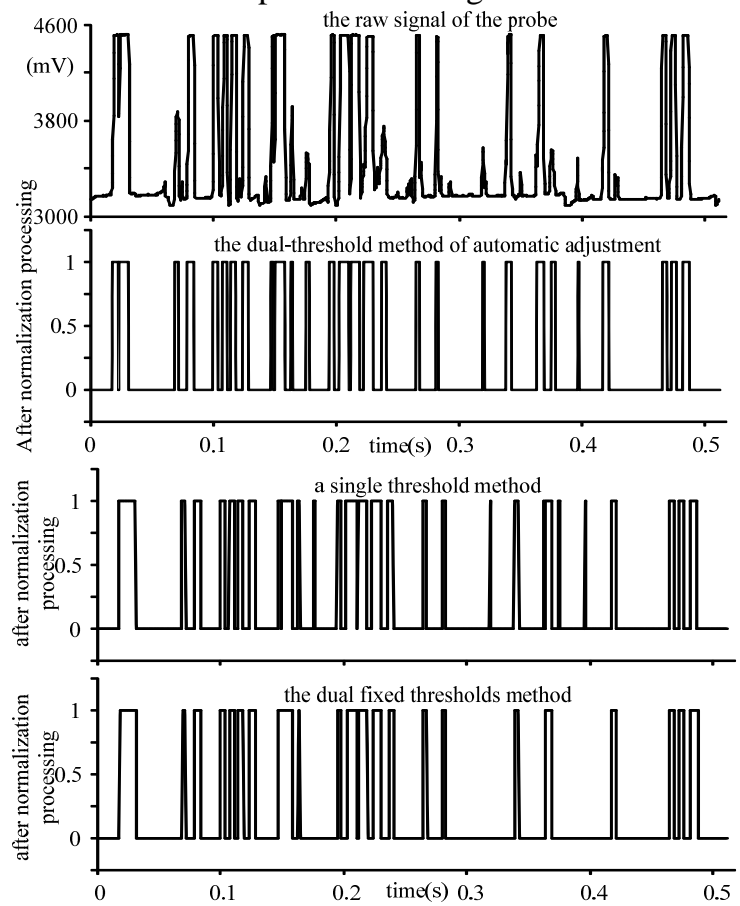

Figure 7. The probe signal and process renderings of total fluid flow with $30 \mathrm{~m}^{3} / \mathrm{d}$

So the single threshold method is a simple algorithm, when the signal has a small amplitude noise interference and threshold variable $p$ is too low, and results in the result larger. When the variable $p$ is too large, although it can filter out noise, it is easy to missing small amplitude signals or bubble edge by using the probe pierced the minute bubble, and results in the result is smaller.

For the fixed dual-threshold method, when the noise is larger, if the low threshold $V_{L}$ is too low, results in the void fraction value serious larger. It will produce small amplitude signals when there are many small bubbles or piercing bubble edge, if the high threshold $V_{H}$ is too high, and results in the void fraction value serious smaller. Therefore, the determinants are more for the high threshold $V_{H}$ and low threshold $V_{L}$ of the fixed dual-threshold, and the setting of the high and low threshold has a certain difficulty.

From the analysis of Fig .7, it can obtain the desired processing effect by using the method of automatic adjustment dual-threshold, detect the signal of probe pierced small bubble or bubble edge, and without considering the influence of noise signal. Large deviation occurred in small-amplitude signal when the threshold value is determined by the minimum and maximum value of signal. The variable threshold $p$ need to consider noise and small signal of piercing bubble edge and other factors.

\section{CONCLUSIONS}

Due to there are no regular patterns about the bubbles in multiphase flow, the diverse sizes and shapes of the bubble, the horizontal radial positions of the bubble pierced by probe has a certain amount of random from the center to edge, caused different signal response characteristics on different horizontal radial positions of probe pierced bubble. In this thesis, processing analogue simulation to the response characteristics of probe pierced bubble by using the modeling ZEMAX, we made a depth analysis both the different horizontal radical positions of probe pierced bubble and the response characteristic of the whole pierced process, it laid good foundation for further study with the mechanism of optical fiber probe sensor pierced bubble, and provided the necessary theory base for the post-processing of the probe signal. We analyzed the applicability and accuracy by using the different threshold methods, and made a reasonable choice of the postprocessing threshold. Then, we can obtain the accurate information of the local void fraction from the probe position, which helps us to learn the distributional identity of gas phase of gas-liquid two-phase flow in the pipeline, can accurately identify and define the video image, which cannot distinguish and boundary flow pattern point.

\section{ACKNOWLEDGMENT}

This work was financially supported by National Science and Technology Major Project of China (NO.2011ZX05020-006).

\section{REFERENCES}

[1] Wegmann A, Melke J, Rohr P R. "Three Phase Liquid-Liquid-Gas Flows in $5.6 \mathrm{~mm}$ and $7 \mathrm{~mm}$ Inner Diameter Piples. ” International Journal of Mutiphase Flow, vol.33, 2007, pp. 484-497.

[2] Woldesemayat M A, Ghajar A J. "Comparison of void fraction correlations for different flow patterns in horizontal and upward inclined pipes,” International Journal of Multiphase Flow, vol.33, 2007, pp. 347-370.

[3] Mena P C, Rocha F A, Teixeira J A, et al. "Measurement of gas phase characteristics using a monofibre optical probe in a threephase flow,” Chemical Engineering Science, vol.63, 2008, pp. 4100-4115.

[4] Xu X, Gong J. "Research Progress of the Oil-Gas-Water Three Phase Flow in Horizontal Pipelines,” Chemical Engineering and Machinery, vol.32, 2005, pp.329.

[5] Tang renhu, Chen tingkuan, Luo yushan, et al. "Void fraction measurement by using optical probes at high temperature and high pressure,“ Journal of Chemical Industry and Engineering (China), vol.52, 2001, pp.560-563.

[6] Angeli P, Hewitt G F. "Flow structure in horizontal oil-water flow,” International Journal of Multiphase Flow, vol.26, 2000, pp. $1117 \sim 1140$.

[7] Van Der Welle R. "Void fraction, bubble velocity and bubble size in two-phase flow," International journal of multiphase flow, vol.11, 1985, pp. 317-345.

[8] Sakamoto A, Saito T. "Robust algorithms for quantifying noisy signals of optical fiber probes employed in industrial-scale practical bubbly flows,” International Journal of Multiphase Flow, vol.41, 2012, pp. 77-90.

[9] Yang Sheng, Luo Yu-shan, Cheng Ting-kuan, et al. "Measurement of void fraction in vertically rising pipes by using optical fiber probes”. Journal of Power Engineering, vol.26, 2006, pp. 875-878.

[10] Lucas G, ZhaoX, Pradhan S. "Optimisation of Four-Sensor Probes for Measuring Bubble Velocity Components in Bubbly Air-Water and Oil-Water Flows," Flow Measurement and Instrumentation, 2011, pp. 50-63.

[11] Ma X, Bi J, Hou X, et al. "Conductively cooled all-solid-state zigzag slab laser,” Chinese Optics Letters, vol.6, 2008, pp. 366-368. 